\title{
Trajectory Planning for Autonomous High-Speed Overtaking using MPC with Terminal Set Constraints
}

\author{
Shilp Dixit \\ Umberto Montanaro \\ and Saber Fallah \\ Mechanical Engineering, University of Surrey \\ Guildford, GU2 7XH, UK \\ Email:s.fallah@surrey.ac.uk
}

\author{
Mehrdad Dianati \\ WMG, University of Warwick \\ Coventry, CV4 7AL, UK
}

\author{
David Oxtoby \\ Tom Mizutani \\ and Alexandros Mouzakitis \\ Jaguar Land Rover Limited \\ Coventry, CV3 4LF, UK
}

\begin{abstract}
With self-driving vehicles being pushed towards the main-stream, there is an increasing motivation towards development of systems that autonomously perform manoeuvres involving combined lateral-longitudinal motion (e.g., lanechange, merge, overtake, etc.). This paper presents a situational awareness and trajectory planning framework for performing autonomous overtaking manoeuvres. A combination of a potential field-like function and reachability sets of a vehicle are used to identify safe zones on a road that the vehicle can navigate towards. These safe zones are provided to a model predictive controller as reference to generate feasible trajectories for a vehicle. The strengths of the proposed framework are: (i) it is free from non-convex collision avoidance constraints, (ii) it ensures feasibility of trajectory, and (iii) it is real-time implementable. A proof of concept simulation is shown to demonstrate the ability to plan trajectories for high-speed overtaking manoeuvres.
\end{abstract}

\section{INTRODUCTION}

The first generation of autonomous cars that demonstrated their ability to perform tasks such as distance maintenance, lane departure warning, etc. helped in improving safety on highways, increase occupant comfort, and reduce the driver workload [1]. However, they still rely on a human driver to either initiate or intervene while performing more challenging manoeuvres (e.g. lane-change, merge, overtake etc.). Overtaking represents a template of such complex manoeuvres as (i) it combines lateral and longitudinal motion of an overtaking vehicle (subject vehicle) while avoiding collisions with a slower moving obstacle vehicle (lead vehicle), and (ii) it includes sub-manoeuvres i.e., lane-changing, lane-keeping, and merging in a sequential manner [2] (see Fig. 1). Hence, the development of autonomous overtaking systems is under great focus since it unlocks the potential to perform a host of different manoeuvres and helps achieve an important milestone towards fully autonomous driving.

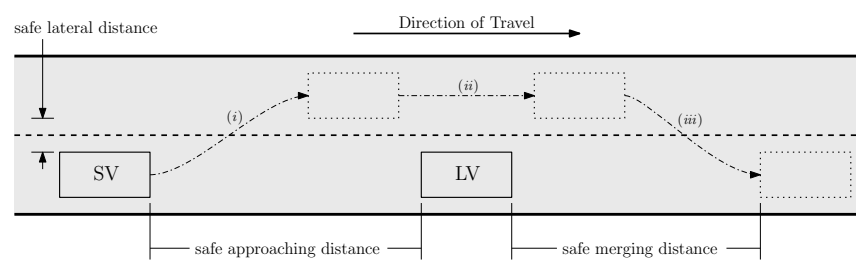

Fig. 1. Schematic of an overtaking manoeuvre (SV: Subject Vehicle, LV: Lead Vehicle)

The inherently intricate structure of overtaking stems from its dependence on a large number of factors such as road condition, weather, traffic condition, participating vehicles, relative velocity, etc. [3]. Furthermore, each overtaking manoeuvre is unique in terms of duration of the manoeuvre, relative velocity between vehicles, etc. [4]-[6] thus making classification and standardisation difficult. There are a variety of diverse ways proposed in literature for planning safe trajectories to perform an autonomous overtaking manoeuvre by treating it as a moving-obstacle avoidance problem. A detailed review of trajectory planning techniques for autonomous overtaking is presented in [7] and some key aspects are discussed below. Incremental search based algorithms such as Rapidly exploring Random Trees (RRT) have been proposed for planning safe trajectories for autonomous overtaking [8]. Even though algorithms incorporating basic vehicle kinematics within a RRT search algorithm have been proposed, the planned trajectories can be jerky which could lead to reduced occupant comfort. Furthermore, the computation times for most search based algorithms are dependent on surrounding traffic density thus making them unsuitable for automotive applications [9]. If accurate knowledge of road and surrounding obstacles is available, potential field based techniques are shown to be successful at generating collision 
free trajectories for avoiding obstacles [10]. However, while guaranteeing collision free trajectories, potential field based methods do not incorporate vehicle dynamics and hence cannot ensure feasibility of the planned trajectory [9]. Model Predictive Control (MPC) helps address these shortcomings with its ability to formulate vehicle dynamics and collision avoidance constraints as a finite-horizon constrained optimisation problem. However, collision avoidance constraints for trajectory planning are generally non-convex which greatly limits the feasibility and uniqueness of the solution to the optimisation problem. Researchers rely on techniques such as convexification [11], change of reference frame [12], and approximation [13] to address the issue. Nevertheless, the optimisation problem formulated by these approaches often require constraint equalities that change in real-time which makes them difficult to implement on a vehicles ECU. In [14] the concept of motion primitives is included within an MPC framework to plan collision avoidance trajectories. However, since these motion primitives are computed offline and accessed via a look-up table, only a subset of all feasible trajectories are considered for motion planning. In [2] overtaking trajectories are generated by directing the vehicle along virtual target points located at safe distances around the LV thus reformulating trajectory planning into a navigation problem. A similar approach inspired from missile guidance systems called Rendezvous Guidance is used to plan a trajectory for an overtaking manoeuvre [15]. However, in all these techniques the SV is modelled as a point mass with no dynamics and hence these methods are unsuitable for highspeed trajectory planning of autonomous vehicles.

In this paper, extracting the relevant benefits of each approach described in the literature, we propose a mathematical framework of potential field-like functions and MPC for performing an autonomous high-speed overtaking manoeuvre. The framework is composed by three components (i) an artificial potential field, (ii) target generation block, and (iii) a trajectory generation block. The artificial potential field is used to map the surrounding region of the SV based on obstacles position, orientation, and relative velocity. At every sampling instant, the target generation block identifies the safest point of the road which is compatible with the dynamics of the SV and computes the reference state set point (e.g., velocity, lateral position, and heading angle) to be tracked. To achieve this aim, the target generation block combines the safe zones in the potential field with the vehicle dynamics capability of the SV which are captured through the reachable set of the SV from its current state. Finally, the trajectory generation block uses an MPC to generate feasible trajectories and steer the vehicle to the required reference (target) states. The MPC approach in [16] is used to solve the reference tracking problem. This MPC method guarantees closed-loop stability while guaranteeing the persistent feasibility of the optimisation problem required by any model predictive control formulation [17]. This paper represents therefore a practical application the theoretical problem formulation presented in [16] to autonomous vehicles.

The paper is structured as follows: Section II introduces the basic symbols and mathematical definitions used in the paper. In Section III, the novel MPC approach in [16] is briefly overviewed to give to the reader the fundamental details of this algorithm which has been used for trajectory planning. In Section IV, the perception system for the vehicle using potential field-like functions is presented, while Section $\mathrm{V}$ is dedicated to the design of the target generation block. The design of trajectory planning based on the MPC method [16] is covered in Section VI. The effectiveness of the framework to support high speed overtraining is numerically shown in Section VII. Finally, the concluding remarks are presented in Section VIII.

\section{MATHEMATICAL Notations AND DEFinitions}

A positive definite matrix $P$ is denoted as $P>0$. For a symmetric matrix $P$ and vector $x,\|x\|_{P}$ denotes the weighted norm given by $\|x\|_{P}=\sqrt{x^{T} P x}$. For vectors $a \in \mathbb{R}^{n_{a}}, b \in \mathbb{R}^{n_{b}}$, vector $(a, b)$ denotes $\left[a^{T}, b^{T}\right]^{T}$. If set $\Gamma \subset \mathbb{R}^{n_{a}+n_{b}}$, then projection operation is defined as $\operatorname{Proj}_{a}(\Gamma)=\left\{a \in \mathbb{R}^{n_{a}}: \exists b \in \mathbb{R}^{n_{b}},(a, b) \in \Gamma\right\}$. For a system with states $x \in \mathcal{X} \subseteq \mathbb{R}^{n_{x}}$ and inputs $u \in \mathcal{U} \subseteq \mathbb{R}^{n_{u}}$ subject to system dynamics described by

$$
\dot{x}=f(x, u)
$$

where $f$ is the state functions (linear or non-linear), one can define the following.

Definition II.1. Reachable Set denoted $\mathcal{R}\left(t_{*} ; x_{0}\right)$ for a system described by (1) is defined as the collection of all states that can be reached at the time instant $t_{*}$ when the initial state is $x(0)=x_{0}$ by applying admissible inputs, i.e.

$$
\mathcal{R}\left(t_{*} ; x_{0}\right)=\bigcup_{u(\cdot), t \in\left[0, t_{*}\right]} x\left(t_{*} ; x_{0}, u(.)\right)
$$

where $u(.) \in \mathcal{U}$ represents the input to system (1) in the time range $\left[0, t_{*}\right]$.

For solving the overtaking problem through the combined use of MPC and potential field, in addition to a coordinate inertial-frame (I-frame), three additional coordinate frames are exploited, i.e., vehicle-frame (V-Frame), obstacle-frame (O-frame), and road-frame ( $\mathrm{R}$-frame). The V-frame is located in the centre of gravity of the SV and follows the RollPitch-Yaw (RPY) convention [18]. Similarly the O-frame is located at the centre of gravity of the LV and follows the RPY 


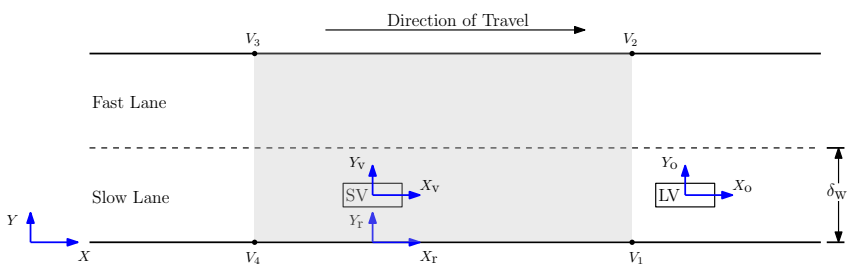

Fig. 2. Road Setup: coordinate frame and range

convention while the road-frame is located at the projection of the origin of vehicle-frame onto the innermost (rightmost) edge of the road with $\mathrm{X}$-axis in the direction of the travel. A generic point on the road is denoted as $w=(X, Y)$, $w_{\mathrm{r}}=\left(X_{\mathrm{r}}, Y_{\mathrm{r}}\right), w_{\mathrm{v}}=\left(X_{\mathrm{v}}, Y_{\mathrm{v}}\right)$, or $w_{\mathrm{o}}=\left(X_{\mathrm{o}}, Y_{\mathrm{o}}\right)$ when expressed in the inertial, road, vehicle, or obstacle frame, respectively. The coordinate frames are depicted in Fig. 2 where $\delta_{\mathrm{w}}$ is the width of the lane while shadow area denotes a rectangle moving along the $\mathrm{R}$-frame with vertices $V=\left\{V_{1}, V_{2}, V_{3}, V_{4}\right\}$. The potential field is computed online within this region for situational awareness in a range relevant for high-speed overtaking. Finally, $T_{j}^{i}$ with $i, j \in\{I, V, R\}$, denotes the linear transformation from $i$-frame to the $j$-frame. Notice that, this transformation can be applied to either individual vectors or sets. When applied to a generic set $\Delta \subset \mathbb{R}^{2}$, $T_{j}^{i}(\Delta)$ denotes the following set $T_{j}^{i}(\Delta) \triangleq\left\{T_{j}^{i}(z)\right\}_{z \in \Delta}$.

\section{MATHEMATICAL BACKGROUND}

This section provides an overview of the MPC approach proposed in [16]. Compared to the classical MPC formulation, the advantage of the control method in [16] is its ability to steer the state of a constrained system toward any set-point whether it belongs or not to the terminal set. The method guarantees the asymptotic convergence of the system state to any admissible target steady state. Furthermore, if the target steady state is not admissible, the control strategy in [16] steers the system to the closest admissible steady state. In the rest of the section, details for implementing this MPC control method are reported.

Given a discrete time LTI system with states $x_{s} \in \mathbb{R}^{n_{x}}$, inputs $u_{s} \in \mathbb{R}^{n_{u}}$, and outputs $y_{s} \in \mathbb{R}^{n_{y}}$, a discrete time state-space system is given by

$$
x_{s}(k+1)=A x_{s}(k)+B u_{s}(k)
$$

where the matrices $A$ and $B$ are constant, it is assumed that the pair $(A, B)$ is stabilisable, and the system subject to hard constraints expressed as,

$$
z=\left(x_{s}, u_{s}\right) \in \mathcal{Z}=\left\{z \in \mathbb{R}^{n_{x}+n_{u}}: A_{z} z \leq b_{z}\right\}
$$

where $A_{z} \in \mathbb{R}^{n_{z} \times\left(n_{x}+n_{u}\right)}$ and $b_{z} \in \mathbb{R}^{n_{z}}$, with $n_{z}$ being the number of constraints, so that $\mathcal{Z}$ is non-empty compact convex polyhedron contain the origin in its interior.
It is noted that the subspace of steady-states and inputs of system (3) have a linear representation of the form

$$
z_{s s}=M_{\theta} \theta
$$

where $z_{s s}=\left(x_{s s}, u_{s s}\right)$ is the stack of the steady-state solution of (3), $x_{s s}$ that is obtained by applying the control action $u_{s s}$ to system (3), and $\theta \in \mathbb{R}^{n_{\theta}}$ is a parameter vector that characterises the subspace of steady-states and inputs and $M_{\theta}$ is a matrix of suitable dimensions. In accordance to [16], given a target steady state $\hat{x}_{s} \in \mathbb{R}^{n_{x}}$, the control objective is to find a control action of the form $u(k)=F_{N}\left(x_{s}(k), \hat{x}_{s}\right)$ such that the state of system (3) is steered as close as possible to the target stare while fulfilling the constraints (4). Furthermore, by denoting $\left\{u_{s}(i), u_{s}(i+1), \ldots, u_{s}(N-1)\right\}$, with $N$ being the prediction horizon, it was proven that the function-map $u(k)=F_{N}\left(x_{s}(k), \hat{x}_{s}\right)$ can be computed by solving the following optimisation problem parametrised in $x_{s}$ and $\hat{x}_{s}$.

$$
\begin{aligned}
& \min _{U_{i}, \theta} V_{N}\left(U_{i}, \theta ; x_{s}, \hat{x}_{s}\right) \\
& \quad \text { subject to } \\
& \quad x_{s}(0)=x_{s} \\
& \quad x_{s}(i+1)=A x_{s}(i)+B u_{s}(i), \quad i=0,1, \ldots, N \\
& \quad\left(x_{s s}, u_{s s}\right)=M_{\theta} \theta \\
& \quad\left(x_{s}(N), \theta\right) \in X_{f}^{g}
\end{aligned}
$$

where the terminal set $\mathcal{X}_{f}^{g}$ is chosen as

$\mathcal{X}_{f}^{g}=\left\{\left(x_{s}, \theta\right) \in \mathbb{R}^{n_{x_{s}}+n_{\theta}}:\left(x_{s}, K x_{s}+L \theta\right) \in \mathcal{Z}, M_{\theta} \theta \in \mathcal{Z}\right\}$

with $K \in \mathbb{R}^{n_{u} \times n_{x}}$ being a constant matrix such that the eigenvalues of $A+B K$ lie within the unit circle, and the cost function $V_{N}\left(U_{i}, \theta, x_{s}, \hat{x}_{s}\right)$ is

$$
\begin{aligned}
V_{N}\left(U_{i}, \theta, x_{s}, \hat{x}_{s}\right)= & \sum_{i=0}^{N}\left[\left\|x_{s}(i)-x_{s s}\right\|_{Q}^{2}+\left\|u_{s}(i)-u_{s s}\right\|_{R}^{2}\right] \\
& +\left\|x_{s}(N)-x_{s s}\right\|_{P}^{2}+\left\|x_{s s}-\hat{x}_{s}\right\|_{T}^{2}
\end{aligned}
$$

where the matrices $Q \in \mathbb{R}^{n_{x} \times n_{x}}, R \in \mathbb{R}^{n_{u} \times n_{u}}, T \in \mathbb{R}^{n_{x} \times n_{x}}$ are positive definite, and $P \in \mathbb{R}^{n_{x} \times n_{x}}$ is a positive definite matrix solving the Lyapunov equation

$$
(A+B K)^{T} P(A+B K)-P=-\left(Q+K^{T} R K\right)
$$

\section{Remarks}

- $U_{i}$ and $\theta$ are the decision variables of the optimisation problem in equation (6), while $x_{s}$ and $\hat{x}_{s}$ are its parameters. Furthermore, the optimal control action is applied using a receding horizon strategy $F_{N}\left(x_{s}, \hat{x}_{s}\right)=u^{*}(0)$, with $u^{*}(0)$ being the first element of the optimal control sequence.

- As the optimisation problem in equation (6) can be expressed as a quadratic programming problem, it can 
be converted to an explicit MPC form to reduce online computations [19].

\section{PERCEPTION}

In this paper, it is assumed that the vehicles are traveling on a two-lane one-way straight road of infinite length. At highway cruising speeds, an overtaking manoeuvre is initiated $\approx$ $50 \mathrm{~m}$ behind the LV and ends $\approx 50 \mathrm{~m}$ in front of it [5]. Hence, the SV needs to have accurate situational awareness of the surrounding obstacles in this range to plan safe trajectories. The authors in [20] mentioned that embedding driving rules and collision avoidance constraints within a multi-objective optimisation problem results in a control laws with large computation requirements. Furthermore, potential field-like functions for environmental perception can be shaped in such a way that it guides towards desired driving behaviour. In this paper the surrounding environment is described through the use of a potential field where several road elements (i.e., road limits, road markers and other road users) are considered for shaping the potential function so as to include driving rules and guide the SV through safe road regions. The net potential function is generated by combining several potential functions where the design of each function is intended to incorporate one or more driving rule(s). The road potential function $\left(U_{\text {road }}\right)$ is designed to keep the $\mathrm{SV}$ away from the road limits, the lane potential function ( $\left.U_{\text {lane }}\right)$ is used for lane-keeping, the lane velocity potential function $\left(U_{\mathrm{vel}}\right)$ is designed such that the SV occupies the innermost (slowest) lane when more than one lane is available, and the car potential function $\left(U_{\text {car }}\right)$ is designed such that a SV either maintains a safe distance to the $\mathrm{LV}$ or if the other lane is available, moves to a faster lane. Similar to the approach from [21], a net potential function $\left(U_{\mathrm{r}}\right)$ is generated by superimposing these individual potential functions to create a perception map that can be used for autonomous overtaking in a human-like manner. The construction of the individual potential functions is discussed below.

\section{A. Lane Velocity Potential}

Different lanes on a road have an implicit velocity associated with them, i.e., the velocity progressively increases from inner (right-most) to outer (left-most) lane. Thus, if one assumes that higher-speeds are represent high-risk, each lane of the road can be appropriated a certain potential to describe its risk. This is achieved by a simple gain based function as shown below

$$
U_{\mathrm{vel}, i}\left(w_{\mathrm{r}}\right)=\gamma\left(v_{\text {lane }, i}\left(w_{\mathrm{r}}\right)-v_{\text {lane }, 1}\left(w_{\mathrm{r}}\right)\right)
$$

where $\gamma$ is a gain factor, $v_{\text {lane }, i}$ is the nominal velocity of the $i^{\text {th }}$ lane, and $U_{\mathrm{vel}, i}$ is the potential due to lane-velocity of the $i^{\text {th }}$ lane.

\section{B. Road Potential}

The road potential [21] is designed such that the boundaries of the road have the highest $(\infty)$ potential and the centre of the road has the lowest potential. A function often used in robotics for perception is used here to describe the lane potential and is given below

$$
U_{\text {road }}\left(w_{\mathrm{r}}\right)=\frac{1}{2} \eta\left(\frac{1}{Y_{\mathrm{r}}-Y_{b}}\right)^{2}
$$

where $\eta$ is a scaling factor and $Y_{b}$ is the y-coordinate of the $b^{\text {th }}$ road edge, $b \in\{1,2\}$.

\section{Lane Potential}

A lane potential function [21] creates a virtual barrier between lanes to direct the SV towards the lane centre. A Gaussian function shown below is used to achieve this desired behaviour.

$$
U_{\text {lane }, i}\left(w_{\mathrm{r}}\right)=A_{\text {lane }} \exp \left(\frac{-\left(Y_{\mathrm{r}}-Y_{1, i}\right)^{2}}{2 \sigma^{2}}\right)
$$

Where $Y_{1, i}$ is the $\mathrm{y}$-coordinate of the $i^{t h}$ lane division, and $\sigma$ and $A_{\text {lane }}$ are scaling factors.

\section{Car Potential}

A technique inspired by [21] is used to embed LV position, orientation, and velocity within the potential function as an obstacle vehicle. By modelling the LV as a rectangular area, virtual triangular wedges, also denoted as buffer zones, are appended to the front and rear of the LV which act as safety buffer zones. The location ( $x, y$ coordinate) of triangles vertex behind the lead-vehicle is calculated based on the velocity of the SV and the headway time $h_{t}$ while the location of the triangles vertex in front of the lead-vehicle is calculated based on the velocity of the LV and the headway time $h_{t}$. By denoting $B_{\mathrm{lv}}$ as the set of coordinates containing the obstacle vehicle and the two triangular wedges, a Yukawa function is used to describe the potential due to an obstacle vehicle as given below

$$
U_{\text {car }}\left(w_{\mathrm{r}}\right)=A_{\text {car }}\left(\frac{e^{-\alpha K_{\mathrm{d}}}}{K_{\mathrm{d}}}\right)
$$

where $\alpha$ is a Yukawa scaling factor, $A_{\text {car }}$ is the Yukawa amplitude [22], and $K_{\mathrm{d}}$ is the Euclidean distance to the nearest coordinate of the obstacle given as

$$
K_{\mathrm{d}}=\min _{b_{0} \in B_{\mathrm{lv}}}\left\|b_{0}-w_{\mathrm{r}}\right\|
$$

These individual potentials are superimposed to obtain an overall perception map in the surrounding of the vehicle given by the expression below.

$$
U_{\mathrm{r}}\left(w_{\mathrm{r}}\right)=U_{\text {vel }}+U_{\text {road }}+U_{\text {lane }}+U_{\text {car }}
$$


Where $U_{\text {lane }}=\sum_{i=1}^{N_{\text {lanes }}} U_{\text {lane }, i}$ and $U_{\text {vel }}=\sum_{i=1}^{N_{\text {lanes }}} U_{\text {vel }, i}$ with $N_{\text {lanes }}$ being the number of lanes. To facilitate trajectory planning the potential field is studied in the inertial frame through the use of the function $U(w) \triangleq U_{\mathrm{r}}\left(T_{\mathrm{I}}^{\mathrm{R}}\left(w_{\mathrm{r}}\right)\right)$. By assigning a threshold limit $U_{\text {safe }}$, the safe regions of the road surrounding the $\mathrm{SV}$ are expressed in the inertial frame using the set

$$
\mathcal{G}=\left\{w \in T_{\mathrm{I}}^{\mathrm{R}}\left(w_{\mathrm{r}}\right): U_{\mathrm{r}}\left(w_{\mathrm{r}}\right) \leq U_{\text {safe }}\right\}
$$

Thus, equation (16) provides a set of safe regions and the SV needs to plan trajectories that keep it within this set thus reducing risk. However, the set (16) does not consider vehicle dynamics of the SV, thus some regions of the road with satisfactory potential may not be reachable in practice. The method designed for selecting reference points in the set of safe regions which are compatible with the dynamics of the SV is detailed in the next section.

\section{Selection of the Target Point}

The dynamics of the SV in the I-frame while driving on a highway at a desired speed $v_{\text {des }}$ are represented with a linear kinematic bicycle model [23]. Hence, by denoting as $x \triangleq\left[X, Y, \psi, v_{\mathrm{x}}\right]^{T} \in \mathcal{X} \subseteq \mathbb{R}^{4}$ the stack of vehicle states, where $\psi$ is the heading angle, $v_{\mathrm{x}}$ is the longitudinal velocity, and $X, Y$ are the longitudinal and lateral displacement of the SV, respectively, the dynamics of the SV are

$$
\dot{x}=A_{\mathrm{c}} x+B_{\mathrm{c}} u
$$

where $u \triangleq\left(a_{\mathrm{x}}, \delta_{\mathrm{f}}\right) \in \mathcal{U} \subseteq \mathbb{R}^{2}$ is the control action with $a_{\mathrm{x}}$ and $\delta_{\mathrm{f}}$ being longitudinal acceleration and front steering angle respectively. The system matrices $A_{\mathrm{c}}$ and $B_{\mathrm{c}}$ are given by

$$
A_{\mathrm{c}}=\left[\begin{array}{cccc}
0 & 0 & 0 & 1 \\
0 & 0 & v_{\mathrm{des}} & 0 \\
0 & 0 & 0 & 0 \\
0 & 0 & 0 & 0
\end{array}\right], B_{\mathrm{c}}=\left[\begin{array}{cc}
0 & 0 \\
0 & 0 \\
0 & v_{\mathrm{des}} / L_{\mathrm{wb}} \\
1 & 0
\end{array}\right]
$$

where $L_{\mathrm{wb}}$ is the SVs wheelbase.

In ideal highway cruising situations, the dynamics of the system can be described by $\dot{x}=\left[v_{\text {des }}, 0,0,0\right]^{T}$ and manoeuvres (e.g., lane-change, merge, etc.) can be thought of as transitions from one set of states to another set of states within the set $\mathcal{X}_{c c}=\{x \in \mathcal{X}: \psi=0\}$. In such ideal scenarios the objective of the $\mathrm{SV}$ is to adjust its trajectory to avoid obstacles while ensuring that the vehicles speed does not exceed the desired longitudinal velocity $v_{\text {des. }}$. Starting from an initial position $w_{0}=\left(X_{0}, Y_{0}\right)$ and traveling at $v_{\text {des }}$, using admissible control actions from the set $\left\{\left(a_{\mathrm{x}}, \delta_{\mathrm{f}}\right): a_{\mathrm{x}} \leq 0,\left(a_{\mathrm{x}}, \delta_{\mathrm{f}}\right) \in \mathcal{U}_{\mathrm{v}}\right\}$, the set $\mathcal{R}_{\text {total }}$ of the states reachable without exceeding the desired velocity $v_{\text {des }}$ in the time interval $t_{*}$ of the system can be computed using equation (2). The set of points on the road that are reachable is a subset of which is given as

$$
\mathcal{R}=\operatorname{Proj}_{w}\left(\mathcal{R}_{\text {total }}\right)
$$

Consequently, the set of reachable lateral and longitudinal coordinates for $\mathrm{SV}$ in the vehicle frame is

$$
\mathcal{R}_{\mathrm{v}}=T_{\mathrm{V}}^{I}(\mathcal{R})
$$

From (16) and (19), the safe zones surrounding the SV which are reachable with respect to the vehicle state and vehicle dynamics is

$$
\mathcal{R}_{\text {safe }} \triangleq \mathcal{G} \cap \mathcal{R}
$$

The, the reference target coordinates $\hat{w}=(\hat{X}, \hat{Y})$ are chosen from $\mathcal{R}_{\text {safe }}$ with the aim to maximise the distance travelled by the $\mathrm{SV}$ in the time interval $t_{*}$, i.e.

$$
\hat{w}=\underset{w \in \mathcal{R}_{\mathrm{safe}}}{\operatorname{argmax}}\left\|w-w_{0}\right\|
$$

Furthermore, it is beneficial that the SV traverses the longitudinal distance from $X_{0}$ to $\hat{X}$ at uniform longitudinal velocity, thus the target velocity $\hat{v}_{\mathrm{x}}$ is selected as

$$
\hat{v}_{\mathrm{x}}=\frac{\left\|\hat{X}-X_{0}\right\|}{t_{*}}
$$

Furthermore, since the $\mathrm{SV}$ is assumed to be traveling on a straight road, the target heading angle of the SV remains $\hat{\psi}=0$. Thus, stacking the reference targets for each state the target state vector for the system $\hat{x}=\left[\hat{X}, \hat{Y}, \hat{\psi}, \hat{v}_{\mathrm{x}}\right]^{T}$ is obtained.

\section{TRAJECTORY GenERATION}

The target states which are generated using the approach in Section $\mathrm{V}$ at each time step result in piecewise references (e.g., if a lane-change is required, $\hat{Y}$ will change from the centre of one lane to another). The MPC approach overviewed in Section III is used in the proposed framework to plan trajectories for directing the vehicle from its current state to a (safe) target state in an admissible way.

As the dynamics of the state $X$ of system (18) depends only on $v_{\mathrm{x}}$, it is possible to further simply the system for the trajectory generation, thus reducing the computational time for its generation. The reduced order system for trajectory generation is

$$
\dot{x}_{\mathrm{r}}=A_{\mathrm{rc}} x_{\mathrm{r}}+B_{\mathrm{rc}} u
$$

where $x_{\mathrm{r}}=\left[Y, \psi, v_{\mathrm{x}}\right]^{T}$ is the system state, $u=\left[a_{\mathrm{x}}, \delta_{\mathrm{f}}\right]^{T}$ is the input, and the system matrices are

$$
A_{\mathrm{rc}}=\left[\begin{array}{ccc}
0 & v_{\mathrm{des}} & 0 \\
0 & 0 & 0 \\
0 & 0 & 0
\end{array}\right], B_{\mathrm{rc}}=\left[\begin{array}{cc}
0 & 0 \\
0 & v_{\mathrm{des}} / L_{\mathrm{wb}} \\
1 & 0
\end{array}\right]
$$


It is noted that system (24) captures the relevant dynamics of the system and is suitable for planning safe and feasible trajectories. Moreover, the discussions in Section V make it evident that the reduced system is in steady-state while the $\mathrm{SV}$ is driving unobstructed on straight highways as $\dot{x}_{\mathrm{r}}(t)=$ $[0,0,0]^{T}$. Furthermore, the reference vector(s) calculated in Section V corresponds to a steady-state condition for system (24) expressed as $x_{\mathrm{r}, \mathrm{ss}}=\left[\hat{Y}, \hat{\psi}, \hat{v}_{\mathrm{x}}\right]^{T}$.

By discretising system (24) with a sampling time $T_{s}$, a discrete time system in the form (3) is obtained with $x_{\mathrm{s}}=x_{\mathrm{r}}, u_{\mathrm{s}}=u$. The set hard constraint $\mathcal{Z}$ in (4) are obtained from the set of states and inputs satisfying the following inequalities given below

$$
\begin{gathered}
x_{\mathrm{r}, \min } \leq x_{\mathrm{r}} \leq x_{\mathrm{r}, \max } \\
u_{\min } \leq u \leq u_{\max }
\end{gathered}
$$

At each discrete time instant $k$ problem in Section III is solved by setting the target state and the initial state as $\hat{x}_{s}=$ $x_{r, s s}$ and $x_{\mathrm{s}}(0)=x_{\mathrm{r}}\left(k \cdot T_{s}\right)$ respectively. Then the reference trajectory $\left[Y^{*}, \psi^{*}, v_{\mathrm{x}}^{*}\right]^{T}$ for $t \in\left[k \cdot T_{s},(k+N) \cdot T_{s}\right]$, with $N$ being the prediction horizon, is computed by applying optimal solution $u^{*}$ to system (18) discretised with the same sampling time $T_{s}$.

The following algorithm summarises all the steps required for performing a safe overtaking manoeuvre in the proposed framework is depicted in the closed loop structure in Fig. 3.

\section{Simulation Results}

In this section, a closed-loop simulation environment is used to evaluate the ability of the proposed framework for planning trajectories for a high-speed overtaking manoeuvre. The scenario is as follows: both the SV and the $\mathrm{LV}$ are traveling on a two-lane one-way road of infinite length at constant longitudinal velocity $v_{\text {des }}$ and $v_{\mathrm{LV}}$ respectively. The dimensions of the road, lane-limits and LVs states are available to the SV on-demand. The desired longitudinal velocity of the SV $v_{\text {des }}$ is provided by the route planner and

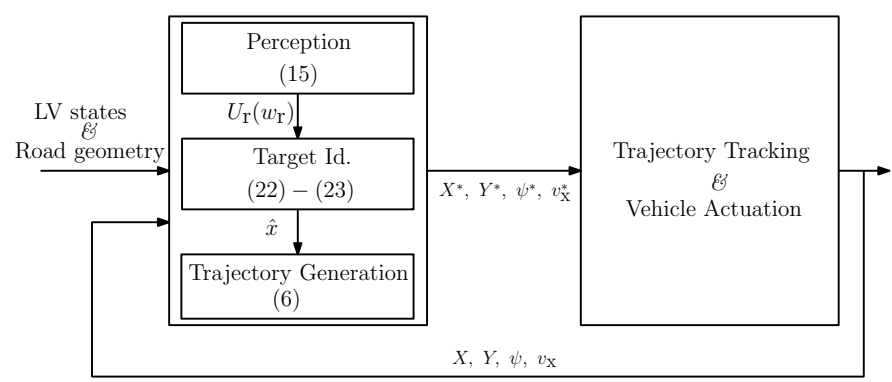

Fig. 3. Closed-loop framework for trajectory planning the left (faster) lane is available. Moreover, it is assumed that a trajectory tracking controller exists on the SV that is able to perfectly track the trajectories planned by the proposed framework. The simulation is performed with the following parameters:

- $v_{\mathrm{des}}=33.33 \mathrm{~m} \mathrm{~s}^{-1} ; L_{\mathrm{wb}}=2.64 \mathrm{~m} ; \delta_{\mathrm{w}}=3.5 \mathrm{~m} ; h_{t}=$ $1.6 \mathrm{~s} ; N_{\text {lanes }}=2$

- $\gamma=2 ; \eta=3 ; A_{\text {lane }}=36 ; \sigma=0.14 \delta_{\mathrm{w}} ; \alpha=0.6$; $A_{\text {car }}=10$

- $x_{s}(0)=\left[\delta_{\mathrm{w}} / 2,0,27.77\right]^{T} ; v_{\mathrm{LV}}(0)=22.22 \mathrm{~m} \mathrm{~s}^{-1}$

- $N=8 ; T_{s}=0.2 \mathrm{~s} ; v_{\mathrm{LV}}=27.77 \mathrm{~m} \mathrm{~s}^{-1} ; t_{*}=1.6 \mathrm{~s}$

- $Q=\operatorname{diag}\left(1 e^{2}, 1 e^{0}, 1 e^{2}\right) ; R=\operatorname{diag}\left(1 e^{1}, 1 e^{0}\right)$

- $K=-R^{-1} B^{T} P ; T=100 P ; P$ is the solution for (9)

- $-\left[0.85,7.6 \cdot 10^{-3}\right]^{T} \leq u \leq\left[0.85,7.6 \cdot 10^{-3}\right]^{T}$

- $-[0,0.035-22.22]^{T} \leq x_{\mathrm{r}} \leq\left[2 \cdot \delta_{\mathrm{w}}, 0.035,36\right]^{T}$

- $V_{1}=(100,0) ; V_{2}=\left(100, N_{\text {lane }} \cdot \delta_{\mathrm{w}}\right) ; V_{3}=$ $\left(-60, N_{\text {lane }} \cdot \delta_{\mathrm{w}}\right) ; V_{4}=(-60,0)$

A simulation environment is initialised with the SV $130 \mathrm{~m}$ behind the LV and the initialisation parameters given above. The simulation is then allowed to run and the proposed framework performs three primary tasks; (i) surrounding perception, (ii) safe target identification, and (iii) trajectory generation at each sampling time. Some details for each task output are given below.

Fig. 4 shows the snapshot of the perception task which is performed during the simulation. The top figure provides a $3 \mathrm{D}$-view of the entire potential function and the local minima at the centre of each lane for guiding a SV can be seen along with the trapezoidal field created by a LV (it is noted that in Fig. 4 large value of the potential field are truncated for the sake of readability of the figure). The bottom plot depicts the level curves for the same time instant in the road-frame. The LV is depicted as red rectangle and the buffer zones (as triangular appendages) where the potential field rapidly increases to prevent the SV from getting too close to the LV during the different phases of an overtaking manoeuvre can be easily observed. The reachable set of the $\mathrm{SV}$ for $v_{\mathrm{des}}=$ $33.33 \mathrm{~m} \mathrm{~s}^{-1}$ and admissible steering inputs is shown in the $\mathrm{V}$ frame in Fig. 5. It is noted that as the precise computation of $\mathcal{R}_{\mathrm{v}}$ according to (2) is not trivial, in the simulation a convex approximation of this set is exploited and the MPT3 toolbox [24] in MATLAB has been used for its computation.

As the region $B_{\mathrm{lv}}$ (unsafe region) surrounding the LV moves in the road-frame with speed $v_{\mathrm{LV}}-v_{\mathrm{x}}$, at each time step the perception of the safe reachable region in (21) and the reference targets in (22) change accordingly. The resulting reference targets for some time steps are depicted in Fig. 6 in the inertial-frame together with the position of the subject and LV. Fig. 6 clear shows the target references selected by the $\mathrm{SV}$ for safely overtaking. For $t<15 \mathrm{~s}$ the SV moves behind the $\mathrm{LV}$, for $t \in[15,30] \mathrm{s}$ the centre of the fast lane has a 


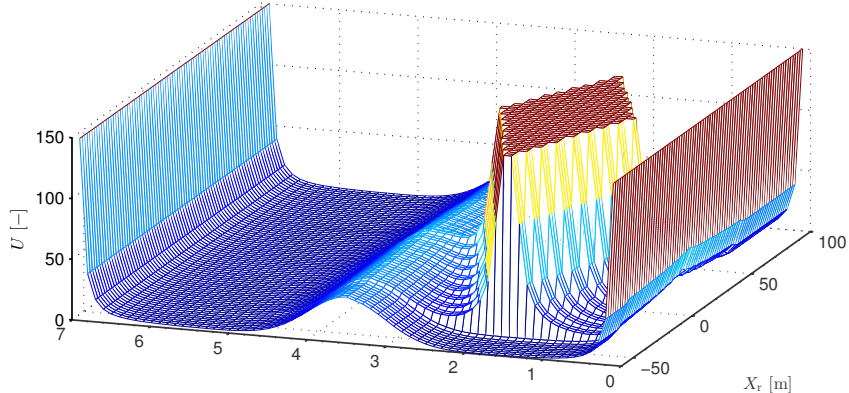

Fig. 4. Cumulative Potential Field $U$

lower potential and it is compatible with the SV dynamics, thus allowing the SV to move to this lane to overtake the LV. Finally for $t>30 \mathrm{~s}$ the centre of the slow lane once again has the lowest potential and is also reachable for the SV. Thus, the SV can merge back in the slow lane at a safe distance in front of the LV thus completing the overtaking manoeuvre.

The reference points dynamically generated are used directly for generating trajectories. The ability of the approach discussed in Section VI to generate feasible trajectories for performing manoeuvres can be observed in Fig. 7 where the planned trajectories and the actual path of the SV are shown in the LVs reference frame (i.e. vehicle-frame). This figure demonstrates that the overtaking manoeuvre is initiated about $50 \mathrm{~m}$ behind the $\mathrm{LV}$ and terminates at a distance over $50 \mathrm{~m}$ in front of the LV thus maintaining the margins expected from a typical highway manoeuvre. These margins are based on the speeds of the two vehicles and the headway time $h_{t}$. Furthermore, the planned trajectories from the MPC controller are stable and do not demonstrate diverging behaviour. By assuming perfect trajectory tracking, the actual trajectory followed by the SV is shown to follow a smooth path from the centre of the slow (right) lane to the fast (left) lane further bolstering the ability of the proposed framework at generating feasible trajectories for performing manoeuvres in structured driving environments. It is noted that in the proposed approach the parameters of the MPC strategy (i.e., $Q, R, P, T$, and $N)$ can be tuned for adjusting the aggressive-

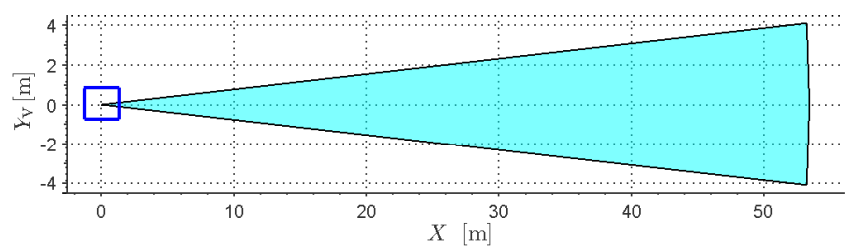

Fig. 5. Reachable subspace $\mathcal{R}_{\mathrm{w}}$ of a vehicle traveling at velocity $v_{\text {des }}$

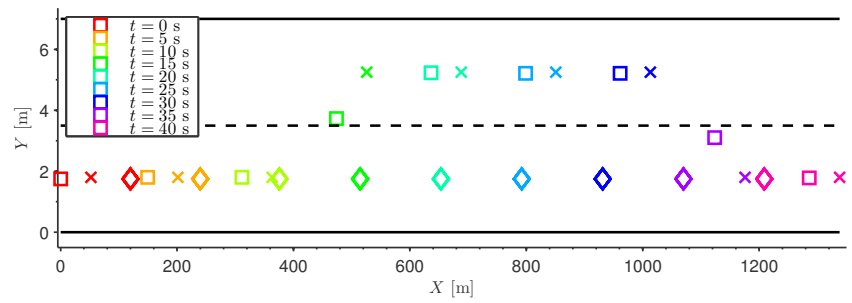

Fig. 6. Reference targets $(\times)$ for different cofigurations of SV $(\square)$ and LV $(\diamond)$ while driving on a highway. Note: solid lines $(-)$ are the road boundaries and dashed line $(--)$ is the lane marking

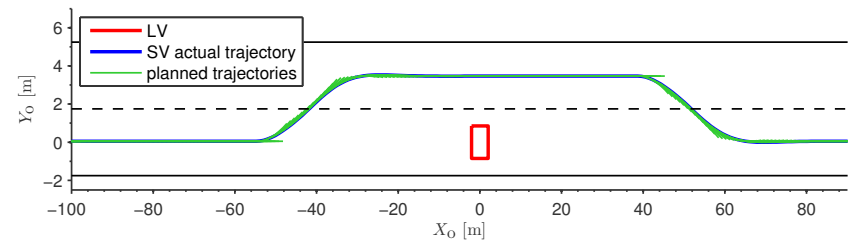

Fig. 7. Simulation Results: planned trajectories and actual trajectory of the $\mathrm{SV}$ during an overtaking manoeuvre in the $\mathrm{LV}$ frame of reference $\left(X_{\mathrm{O}}, Y_{\mathrm{O}}\right)$

ness of a manoeuvre. Additionally, the trajectories planned by this novel MPC are persistently feasible thus providing an additional safety-net to the autonomous driving functionality.

Finally, the trajectories of the subject and LV are shown two vehicles and the relevant states and inputs of the SV are shown in in the inertial frame in Fig. 8. The top plot shows the actual path followed by the two vehicles and the trajectory of the overtaking manoeuvre for the SV can be observed. Furthermore, since the SV is travelling with a higher longitudinal velocity it covers a larger portion of the road segment in the given time. The response of the MPC controller while; (i) increasing the longitudinal velocity of the SV, (ii) providing steering action for performing the lateral motions, (iii) providing smooth inputs, and (iv) respecting the system constraints can be observed in the remaining plots of Fig. 8.

\section{CONCLUSION AND Future Work}

In this paper, a mathematical framework for trajectory planning for an autonomous high-speed driving was presented. The components of this proposed framework were; (i) artificial potential fields were used to map the surrounding region of an autonomous vehicle, (ii) target generation block to identify safe target regions surrounding the SV based on a reachability analysis, and (iii) an asymptotically stable MPC based controller to plan feasible trajectories for the SV to direct it along the safe regions of the road. Thus, in this framework the onus of collision avoidance lies with the perception sub-system and the onus of feasible trajectory 

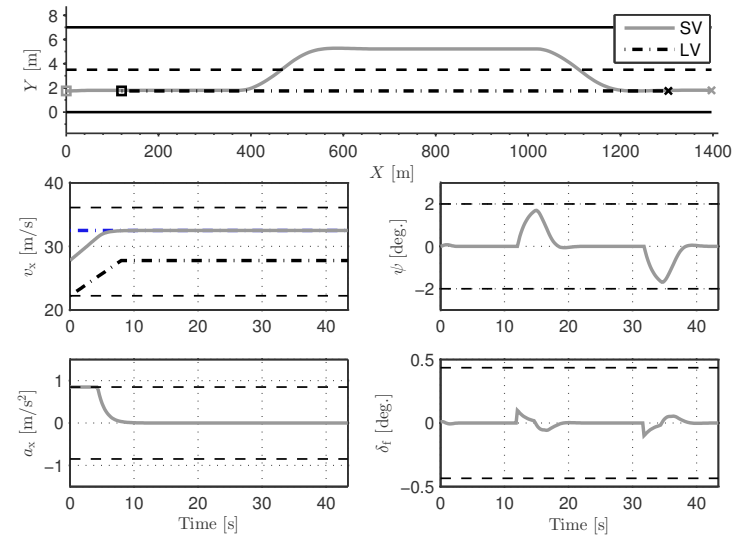

Fig. 8. Simulation Results: SV and LV trajectories, longitudinal velocity, heading angle, longitudinal acceleration, and steering angle for a high-speed overtaking manoeuvre. Note: $(--)$ are the system constraints, $v_{\text {des }}$ shown by blue line

lies with the MPC controller. This modular design allows the framework to avoid non-convex constraints allowing for an MPC formulation that can be converted to an explicit control law using common multi-parametric programming tools. Numerical results demonstrated that the algorithm is able to respect the safety considerations for high speed overtaking manoeuvre and generate trajectories which are also compatible with the vehicle dynamics and safety considerations.

Future work includes enhancing the MPC controller to generate feasible trajectories over a larger longitudinal velocity range by making it robust against non-linear lateral dynamics. Finally, the framework will be extend to consider multiple moving obstacles within the perception and target generation components.

\section{ACKNOWLEDGMENT}

This work was supported by Jaguar Land Rover and the UK-EPSRC grant EP/N01300X/1 as part of the jointly funded Towards Autonomy: Smart and Connected Control (TASCC) Programme and is subject to UK patent under UKIPO GB1801968.7 (under review).

\section{REFERENCES}

[1] A. Eskandarian, Handbook of Intelligent Vehicles: Vol 2. Springer, 2012.

[2] P. Petrov and F. Nashashibi, "Modeling and Nonlinear Adaptive Control for Autonomous Vehicle Overtaking," IEEE Transactions on Intelligent Transportation Systems, vol. 15, no. 4, pp. 1643-1656, 2014.

[3] B. Vanholme, D. Gruyer, B. Lusetti, S. Glaser, and S. Mammar, "Highly automated driving on highways based on legal safety," IEEE Transactions on Intelligent Transportation Systems, vol. 14, no. 1, pp. 333-347, 2013.
[4] J. Baber, J. Kolodko, T. Noel, M. Parent, and L. Vlacic, "Cooperative autonomous driving: intelligent vehicles sharing city roads," IEEE Robotics \& Automation Magazine, vol. 12, no. 1, pp. 44-49, 2005.

[5] G. Hegeman, K. Brookhuis, and S. Hoogendoorn, "Opportunities of advanced driver assistance systems towards overtaking," EJTIR, vol. 5, no. 4, pp. 281-296, 2005.

[6] E. I. Vlahogianni, "Modeling duration of overtaking in two lane highways," Transportation Research Part F: Traffic Psychology and Behaviour, vol. 20, pp. 135-146, 2013.

[7] S. Dixit, S. Fallah, U. Montanaro, M. Dianati, A. Stevens, F. Mccullough, and A. Mouzakitis, "Trajectory planning and tracking for autonomous overtaking: State-of-the-art and future prospects," Annual Reviews in Control, vol. 45, pp. 76-86, 2018.

[8] L. Ma, J. Xue, K. Kawabata, J. Zhu, C. Ma, and N. Zheng, "A fast RRT algorithm for motion planning of autonomous road vehicles," in 2014 17th IEEE International Conference on Intelligent Transportation Systems, ITSC 2014, 2014, pp. 1033-1038.

[9] C. Katrakazas, M. Quddus, W.-H. Chen, and L. Deka, "Real-time motion planning methods for autonomous on-road driving: State-ofthe-art and future research directions," Transportation Research Part C: Emerging Technologies, vol. 60, pp. 416-442, 2015.

[10] S. Kitazawa and T. Kaneko, "Control Target Algorithm for Direction Control of Autonomous Vehicles in Consideration of Mutual Accordance in Mixed Traffic Conditions," in International Symposium on Advanced Vehicle Control 2016, 2016.

[11] G. Franze and W. Lucia, "A Receding Horizon Control Strategy for Autonomous Vehicles in Dynamic Environments," IEEE Transactions on Control Systems Technology, vol. 24, no. 2, pp. 695-702, 2016.

[12] N. Murgovski and J. Sjöberg, "Predictive cruise control with autonomous overtaking," in IEEE 54th Annual Conference on Decision and Control (CDC), 2015, 2015, pp. 644-649.

[13] J. Nilsson, P. Falcone, M. Ali, and J. Sjöberg, "Receding horizon maneuver generation for automated highway driving," Control Engineering Practice, vol. 41, pp. 124-133, 2015.

[14] A. Gray, Y. Gao, T. Lin, J. K. Hedrick, H. E. Tseng, and F. Borrelli, "Predictive Control for Agile Semi-Autonomous Ground Vehicles using Motion Primitives," University of California, Berkeley, Berkeley, Tech. Rep., 2012.

[15] U. Ghumman, F. Kunwar, and B. Benhabib, "Guidance-Based On-Line Motion Planning For Autonomous Highway Overtaking," International Journal On Smart Sensing And Intelligent Systems, vol. 1, no. 2, pp. 549-571, 2008.

[16] D. Limón, I. Alvarado, T. Alamo, and E. F. Camacho, "MPC for tracking piecewise constant references for constrained linear systems," Automatica, vol. 44, no. 9, pp. 2382-2387, 2008.

[17] J. M. Maciejowski, Predictive control: with constraints. Pearson education, 2002.

[18] T. D. Gillespie, Fundamentals of Vehicle Dynamics, 1997.

[19] T. Besselmann and M. Morari, "Autonomous vehicle steering using explicit LPV-MPC," 2009 European Control Conference, no. 1, pp. 2628-2633, 2009.

[20] E. Semsar-Kazerooni, J. Verhaegh, J. Ploeg, and M. Alirezaei, "Cooperative adaptive cruise control: An artificial potential field approach," in 2016 IEEE Intelligent Vehicles Symposium (IV), 2016, pp. 361-367.

[21] M. T. Wolf and J. W. Burdick, "Artificial potential functions for highway driving with collision avoidance," in Proceedings - IEEE International Conference on Robotics and Automation, 2008, pp. 37313736.

[22] R. Volpe and P. Khosla, "Manipulator Control with Superquadric Artificial Potential Functions: Theory and Experiments," IEEE Transactions on Systems, Man and Cybernetics, vol. 20, no. 6, pp. 1423-1436, 1990.

[23] R. Rajamani, "Lateral vehicle dynamics," in Vehicle Dynamics and Control, 2012, ch. 2, pp. 15-46.

[24] M. Herceg, M. Kvasnica, C. Jones, and M. Morari, "Multi-Parametric Toolbox 3.0," in Proceedings of the European Control Conference, 2013, pp. 502-510. 